\title{
Occupational Stress versus Cortisol Associated with Psychological Variables and Gender Differences among Women and Men in Nursing
}

\author{
Virginia Dresch 1,2,3, María del Pilar Sánchez-López ${ }^{1,2,4}$, Ana Isabel Saavedra1,2,5 \\ ${ }^{1}$ EPSY-Research Group Psychological Styles, Gender, and Health, Madrid, Spain \\ ${ }^{2}$ Red Hygeia-Health \& Gender International Alliance, Madrid, Spain \\ ${ }^{3}$ Universidade Federal Fluminense, Rio de Janeiro, Brazil \\ ${ }^{4}$ Universidad Complutense de Madrid, Madrid, Spain \\ ${ }^{5}$ Hospital Universitario de Getafe, Madrid, Spain \\ Email: virginiadresch@vm.uff.br
}

Received 11 June 2015; accepted 11 July 2015; published 14 July 2015

Copyright (C) 2015 by authors and Scientific Research Publishing Inc.

This work is licensed under the Creative Commons Attribution International License (CC BY). http://creativecommons.org/licenses/by/4.0/

\section{(c) (i) Open Access}

\section{Abstract}

The present research focused on a sample of Spanish male and female nurses to determine whether psychological variables are related to occupational stress and cortisol. Spanish male $(n=98)$ and female $(n=98)$ nurses, matched in diverse sociodemographic variables, completed measures of occupational stress, cortisol 8 am $(8 \mathrm{~h})$, and cortisol 8 pm $(20 \mathrm{~h})$, and psychological variables. Multivariable regression analyses revealed different patterns of association among the variables when analyzing subjective and (occupational stress) objective indicators (cortisol at $8 \mathrm{~h}$ and cortisol at $20 \mathrm{~h}$ ). In male nurses, higher stress is related to worse self-perceived health, worse mental health, less cortisol measured at $8 \mathrm{~h}$, and more morningness. For the female nurses, higher stress is related to being younger, worse self-perceived health, and less job satisfaction. The only similarities found in the target variables and the measures of stress studied in the groups of males and females are worse self-perceived health and worse mental health associated with more occupational stress.

\section{Keywords}

Occupational Stress, Cortisol, Health, Nursing, Spain 


\section{Introduction}

The relations between objective measures (cortisol) and subjective measures of occupational stress and their corresponding impact on health are still unclear. Physical and/or psychological stress activates the Sympathetic Nervous System, which increases levels of plasma cortisol and hyperarousal of the hypothalamic-pituitaryadrenal (HPA) axis, which also increases cortisol levels, the most widely used glucocorticoid (followed by dehydroepiandrosterone [DHEA]) to measure the physiological response of organisms to stressful stimuli [1].

Almeida, Piazza, and Stawski [2] find in both sexes an increase in the levels of morning cortisol with age, and of other inter- and intra-individual variables that daily modify the levels of this hormone. The studies carried out by Kajantie and Phillips [3] and Deane, Chummun, and Prashad [4] with male and female nurses also describe how levels of cortisol increase with age, and conclude that the waking response to cortisol is higher in morningness individuals, independently of sex and of the woman's menstrual phase.

Although more stress is expected to relate to higher plasma cortisol, Harris, Ursin, Murison, and Eriksen [5] found no significant relations between cortisol and job stress, concluding that only decision authority and coffee were significantly related to cortisol levels in the evening. The inverse relation, that is, low waking levels of cortisol related to high levels of chronic stress and chronic fatigue in general population has also been reported [6] as well as high burnout scores in manual workers [7], professional nursing staff [5] [8] and professional teaching staff [9].

Wang, Korczykowski, Rao, Fan, Pluta, Gur, McEwen, and Detre [10] reported slight increases in levels of plasma cortisol in men compared with women, although they found clear differences using neuroimaging (nuclear magnetic resonance [NMR]) in psychological responses to stressors and their impact on the brain.

The research of Kajantie and Phillips [3] and the review carried out by Kudielka and Kirschbaum [11] reported that levels of plasma cortisol increase with acute stress in both sexes, but the triggers for this response are different for men and women.

Lastly, the study of Bergman, Ahmad, and Stewart [12] with male and female doctors indicates that the variables related to the number of hours both sexes dedicate to housework and childcare show that a higher perception of family responsibilities is related to higher levels of evening cortisol. In nurses, Eriksen, Tambs, and Knardahl [13] find that work factors explain a modest part of the psychological distress in nurses' aides. Exposure to role conflicts and threats and violence at work may contribute to psychological distress in nurses' aides.

There are many studies in the literature conducted with women and a few studies with men. For example, Hsu, Chen, Yu, and Lou [14] find that job stress of male nurses was strongly correlated with occupational burnout. Variables predicting career and life satisfaction among male nurses included perceptions of gender-related work barriers, conflict between work and family, social support from significant others, confidence in job skills, and being comfortable about physical and emotional expression with other men [15]. In addition, very few studies include homogeneous samples of men and women that are comparable in number and variables such as civil status, family burdens, or the units where they work, in order to analyze differences between male and female nurses, as reported in the study of [16]. It is therefore difficult to reach conclusions based on the differences between male and female nurses in the variables of this study.

Therefore, on the basis of these facts, the goal of this study was to verify the relations between occupational stress (objective and subjective measures) and psychological variables in male and female nurses. Specifically, we propose to determine the existence of: a) sex differences in the target variables (age, perceived health, mental health, job satisfaction, occupational stress, cortisol $8 \mathrm{~h}$, cortisol $20 \mathrm{~h}$, and morningness-eveningness), using a group of male and female participants balanced in number and paired by the authors in sociodemographic variables; b) differences in the correlations between objective measures (cortisol 8h and cortisol $20 \mathrm{~h}$ ) and subjective measures of occupational stress and the target variables; and c) differences in the equations of cortisol (objective measure of stress) and occupational stress (subjective measure of stress) with regard to the target variables.

\section{Methods}

\subsection{Participants}

As we propose to compare health behavior of male and female nurses, and given the shortage of male nurses, which may skew the characteristics, we have taken particular care in choosing and matching the group of male 
and female participants.

From a total number of 103 male nurses in one of the tertiary university hospitals of the Community of Madrid (representative of the hospitals in the network of the Spanish National Health system), 98 nurses volunteered to participate in the study. In order to obtain two equivalent groups, nurses were chosen by matching them in the most important sociodemographic and work variables from the initial survey of nurses. Of the total number of female hospital nurses (588), 98 were selected, as they met the same sociodemographic criteria (age, marital status, number of children and number of dependents they cared for), and with the same work shift and service in the hospital as their male colleagues.

Nurses' average age was 32.45 years $(S D=8.42)$, ranging between 20 and 56 . The males' average age was 32.29 years $(S D=8.64)$ and females' was $32.60(S D=8.20)$, ranging between 22 and 56 years for females, and 20 and 56 for males. The males had worked for an average of 101.92 months $(S D=99.43)$ and the females 114.92 months $(S D=92.83)$.

Men and women who were taking corticoids and/or pregnant women were excluded from the study at the time of the individual interview because, in both cases, levels of plasma cortisol may be altered.

\subsection{Instruments}

- Sociodemographic variables were assessed with a questionnaire. We considered age, marital status, having children, having elderly people to care for, the service where they worked, whether they were on night shift, and whether they received any help with housework.

- To measure perceived health, occupational stress, and perceived job satisfaction, we used the correspond items from the National Health Survey [17].

- Cortisol: As cortisol is a hormone segregated by circadian rhythm [18], we performed two extractions of plasma cortisol, one at $8 \mathrm{~h}$ and one at $20 \mathrm{~h}$ on the same day.

- Mental Health: The Spanish version [19] of the 12-Item General Health Questionnaire (GHQ-12) [20] was used to measure mental health.

- Morningness/Eveningness: we administered the Spanish version [21] of the Composite Scale [22].

\subsection{Procedure}

This study obtained approval from the institutional review board of the university hospital in which the participants work (Madrid, Spain). The hospital is representative of the network of Spanish Public Health hospitals. All participants were invited individually to take part in the study after hearing a short description of the nature of the study. All participants gave (written and signed) informed consent after the researchers had explained the purpose of the investigation and a description of the procedures of the study, guaranteeing participants' freedom to withdraw from any part of the study without consequences. Anonymity of their data was guaranteed.

After signing informed consent, participants completed the questionnaires presented in the Instruments section. At that time, the date was set to extract the two samples of plasma cortisol, at $8 \mathrm{~h}$ and at $20 \mathrm{~h}$ on the same day, selecting a day that coincided with the participant's work day (daytime or in the evening), providing they had not worked the night immediately preceding the extraction (that is, they should have rested the previous night). They were requested to fast, not smoke or perform any physical exercise before the extraction.

\subsection{Data Analysis}

Preliminary analyses comparing ages, perceived health, mental health, job satisfaction, occupational stress, cortisol $8 \mathrm{~h}$, cortisol $20 \mathrm{~h}$, and morningness-eveningness between the male/female was conducted. Secondly, the correlations between the objective (cortisol $8 \mathrm{~h}$ and cortisol $20 \mathrm{~h}$ ) and subjective measures (occupational stress) of stress and the remaining variables (age, perceived health, mental health, job satisfaction and morningnesseveningness) were calculated. Thirdly, stepwise linear regression was conducted to determine the set of health variables most closely related to stress. The $R^{2}$ statistic was used to study goodness of fit, and the $F$-test for over all fit of the regression models.

\section{Results}

No significant differences were found in age, marital status, number of children, dependents to care for, and 
working on night shift between females and males (see Table 1). Males and females only differed significantly in help with housework $\left(\chi^{2}=5.84 ; p<0.05\right)$, in which $41.8 \%$ of women versus $25.5 \%$ of men received this kind of support at home.

Females perceived worst health $(t=-2.49, p<0.05)$ and more occupational stress $(t=-2.17, p<.05)$ than males (see Table 2). According to Cohen's (1988) guidelines, the magnitude of these differences was low.

Perceived health and mental health were significantly and directly correlated to occupational stress in the total sample, and in males and females (see Table 3). Effect sizes of both variables were medium in the total sample and in males, and low in perceived health and medium in mental health in females. Other variables (job satisfaction and morningness-eveningness) were inversely correlated to occupational stress, but only in the total sample and in females, with lower effect sizes except for job satisfaction, which was medium. Concerning cortisol measured at $8 \mathrm{~h}$ and at $20 \mathrm{~h}$, age was inversely correlated in the total sample, and both in the female and male samples. This correlation was statistically significant for $8 \mathrm{~h}$ cortisol in the entire sample and in females. Occupational stress was also inversely correlated to cortisol at $8 \mathrm{~h}$, but only in males. In addition to being related to cortisol at $8 \mathrm{~h}$, cortisol measured at $20 \mathrm{~h}$ was only inversely correlated with morningness-eveningness for the entire sample and for females.

All variables included in each of the multivariate regression models (see Tables 4-6) met the assumption of non-multicolinearity (that is, tolerance values higher than 0.80 ).

The first multivariable regression model (see Table 4) indicated significant variables related to occupational

Table 1. Sociodemographic characteristics of the participants.

\begin{tabular}{|c|c|c|}
\hline Female $(n=98)$ & Male $(n=98)$ & Statistic ( $p$ values) \\
\hline Age & Age & \\
\hline$M(S D)=32.60(8.20)$ & $M(S D)=32.29(8.63)$ & $t=-0.26(\mathrm{~ns})$ \\
\hline Range $=22-56$ & Range $=20-56$ & \\
\hline Marital status & Marital status & \\
\hline 66.3 single & 66.3 single & $\chi^{2}=0.00(n s)$ \\
\hline $29.6 \%$ married/de facto marriage & $29.6 \%$ married/de facto marriage & \\
\hline 3.1 separated or divorced & 3.1 separated or divorced & \\
\hline $1.0 \%$ widowed & $1.0 \%$ widowed & \\
\hline Number of children & Number of children & \\
\hline $74.5 \%$ none & $74.5 \%$ none & $\chi^{2}=0.74(n s)$ \\
\hline $9.2 \%$ one & $12.2 \%$ one & \\
\hline 16.3 two & 13.3 two & \\
\hline Dependents to care for & Dependents to care for & \\
\hline 5.1 yes & 3.1 yes & $\chi^{2}=0.17(n s)$ \\
\hline 94.9 no & 96.9 no & \\
\hline Night shift & Night shift & \\
\hline 77.6 yes & 78.6 yes & $\chi^{2}=0.03(n s)$ \\
\hline 22.4 no & 21.4 no & \\
\hline Help with housework & Help with housework & \\
\hline $41.8 \%$ yes & 25.5 yes & \\
\hline 58.2 no & 74.5 no & \\
\hline
\end{tabular}

${ }^{*} p<0.05 . n s=$ nonsignificant. 
Table 2. Means and standard deviations of stress and health variables, $t$ results and effect size of the differences.

\begin{tabular}{|c|c|c|c|c|c|}
\hline & $\begin{array}{l}\text { Total }^{\mathrm{a}} \\
\mathrm{M} \text { (SD) }\end{array}$ & $\begin{array}{c}\text { Male Nurses }^{b} \\
\text { M (SD) }\end{array}$ & $\begin{array}{c}\text { Female Nurses }^{c} \\
\text { M (SD) }\end{array}$ & $t$ & effect size \\
\hline Perceived health & $1.93(0.60)$ & $1.83(0.51)$ & $2.04(0.67)$ & $-2.49^{*}$ & low \\
\hline Mental health & $10.68(4.64)$ & $10.09(4.00)$ & $11.27(5.15)$ & $-1.79 \mathrm{~ns}$ & - \\
\hline Job satisfaction & $5.34(1.27)$ & $5.40(1.32)$ & $5.29(1.23)$ & $0.61 \mathrm{~ns}$ & - \\
\hline Occupational stress & $5.02(1.32)$ & $4.82(1.38)$ & $5.22(1.24)$ & $-2.17^{*}$ & low \\
\hline Cortisol 8h & $18.58(7.15)$ & $17.70(5.14)$ & $19.46(8,64)$ & $-1.72 \mathrm{~ns}$ & - \\
\hline Cortisol 20h & $7.24(5.31)$ & $7.40(5.65)$ & 7.08 (4.98) & $0.42 \mathrm{~ns}$ & - \\
\hline Morningness/eveningness & $32.93(6.67)$ & $33.21(6.65)$ & $32.66(6.70)$ & $0.57 \mathrm{~ns}$ & - \\
\hline
\end{tabular}

${ }^{\mathrm{a}} \mathrm{N}=196 .{ }^{\mathrm{b}} n=98 .{ }^{c} n=98 .{ }^{*} p<0.05$.

Table 3. Pearson's correlations between objective and subjective stress measures and health variables in total sample, males and females, respectively.

\begin{tabular}{ccccccccccccc}
\hline & \multicolumn{3}{c}{ Occupational stress } & \multicolumn{3}{c}{ Cortisol $8 \mathrm{~h}$} & \multicolumn{3}{c}{ Cortisol $20 \mathrm{~h}$} \\
\hline & Total & Males & Females & Total & Males & Females & Total & Males & Females \\
\hline Age & -0.10 & 0.00 & $-0.24^{*}$ & $-0.19^{* *}$ & -0.10 & $-0.26^{* *}$ & -0.12 & -0.09 & -0.16 \\
Perceived health & $0.30^{* *}$ & $0.37^{* *}$ & $0.22^{*}$ & 0.00 & 0.14 & -0.10 & -0.03 & 0.04 & -0.10 \\
Mental health & $0.34^{* *}$ & $0.34^{* *}$ & $0.32^{* *}$ & 0.05 & 0.09 & 0.01 & 0.07 & 0.01 & -0.13 \\
Job satisfaction & $-0.22^{* *}$ & -0.10 & $-0.35^{* *}$ & 0.03 & -0.02 & 0.08 & 0.04 & 0.01 & 0.09 \\
Occupational stress & - & - & - & 0.00 & $-0.20^{*}$ & 0.11 & -0.01 & -0.08 & 0.07 \\
Cortisol 8 h & 0.00 & $-0.20^{*}$ & 0.11 & - & - & - & $0.19^{* *}$ & 0.15 & $0.24^{*}$ \\
Cortisol 20 h & -0.01 & -0.08 & 0.07 & $0.19^{* *}$ & 0.15 & $0.24^{*}$ & - & - & - \\
Morningness-eveningness & $-0.20^{* *}$ & -0.16 & $-0.23^{*}$ & 0.02 & 0.04 & 0.02 & $-0.21^{* *}$ & $-0.29^{* *}$ & -0.13 \\
\hline
\end{tabular}

${ }^{* *} p<0.01 .{ }^{*} p<0.05$. Effect size: $r>0.10$ (low), $r>0.30$ (medium), $r>0.50$ (high).

Table 4. Factors related to the level of occupational stress. Final stepwise, linear regression equation.

\begin{tabular}{cccccccccccc}
\hline & \multicolumn{3}{c}{ Total sample } & \multicolumn{3}{c}{ Males } & \multicolumn{3}{c}{ Females } \\
\hline & $\beta$ & $t$ & $P$ & $\beta$ & $t$ & $p$ & $\beta$ & $t$ & $p$ \\
Age & - & - & - & - & - & - & -0.27 & -3.01 & 0.003 \\
Perceived health & 0.22 & 3.28 & 0.001 & 0.32 & 3.51 & 0.001 & 0.20 & 2.12 & 0.036 \\
Mental health & 0.28 & 4.06 & 0.000 & 0.27 & 2.91 & 0.004 & - & - & - \\
Job satisfaction & - & - & - & - & - & - & -0.31 & -3.37 & 0.001 \\
Cortisol 8 h & - & - & - & -0.27 & -3.06 & 0.003 & - & - & - \\
Morningness-eveningness & - & - & - & - & - & - & - & - & - \\
\hline
\end{tabular}

$R^{2}$ the entire sample: 0.16 (explains 16\% of the variance). $R^{2}$ males: 0.25 (explains $25 \%$ of the variance). $R^{2}$ females: 0.20 (explains $20 \%$ of the variance).

stress, except for the morningness-eveningness. The equations are different for men and women, except in the case of perceived health, which was significant for both groups, although it was higher in men. In the group of men, mental health $(\beta=0.27, p<0.001)$ and cortisol at $8 \mathrm{~h}(\beta=-0.27, p<0.001)$ were also related to occupational stress. In addition to perceived health $(\beta=0.20, p<0.01)$, in the group of females, age $(\beta=-0.27, p<$ 
$0.01)$ and job satisfaction $(\beta=-0.31, p<0.001)$ were related, albeit negatively.

The second multivariate regression model (see Table 5 ) revealed some variables with significant relation with cortisol $8 \mathrm{~h}$. Age was negatively related to cortisol $8 \mathrm{~h}$ in the total sample $(\beta=-0.19, p<0.01)$ and in females $(\beta$ $=-0.26, p<0.01)$; the relation with perceived health was positive $(\beta=0.25, p<0.05)$, and with occupational stress $(\beta=-0.29, p<0.01)$, negative, but only in males.

The third multivariate regression model (see Table 6) indicated only one variable (morningness-eveningness) significantly related to cortisol $20 \mathrm{~h}$ in the total sample $(\beta=-0.20, p<0.01)$ and in the males $(\beta=0.28, p<$ $0.01)$.

\section{Discussion}

The goal of this study was to analyze the relations between occupational stress, cortisol and psychological variables and gender differences in a sample of male and female nurses. Firstly, it is important to underline that when comparing the group of male and female nurses in the target variables, we only found significant differences-albeit with a low effect size-in self-perceived health and occupational stress (female nurses report slightly poorer self-perceived health and more occupational stress than male nurses). Pairing the two groups in the sociodemographic variables studied (age, educational level, marital status, number of children, dependent people to care for, night shift) produces significant differences in the psychological variables of interest (perceived health, mental health, occupational stress, job satisfaction, and morningness-eveningness), independently of whether such differences are minimal or insignificant, corroborating the results of other studies e.g., [23]-[25]. That is, both male/female and gender are very important variables to consider when analyzing the differences produced in the target variables, but so is age, job situation, educational level, living with a partner, living in a rural or urban environment, etc. These variables can be as important as or more so than the fact of being male or female.

We found a differential pattern of associations among the psychological variables and type of measurement of

Table 5. Factors related to the level of cortisol $8 \mathrm{~h}$. Final stepwise, linear regression equation.

\begin{tabular}{cccccccccccc}
\hline & \multicolumn{3}{c}{ Total sample } & \multicolumn{3}{c}{ Males } & \multicolumn{3}{c}{ Females } \\
& $\beta$ & $t$ & $P$ & $\beta$ & $t$ & $p$ & $\beta$ & $t$ & $p$ \\
\hline Age & -0.19 & 2.73 & 0.007 & - & - & - & -0.26 & -2.71 & 0.008 \\
Perceived health & - & - & - & 0.25 & 2.40 & 0.018 & - & - & - \\
Mental health & - & - & - & - & - & - & - & - & - \\
Job satisfaction & - & - & - & - & - & - & - & - & - \\
Occupational stress & - & - & - & -0.29 & -2.79 & 0.006 & - & - & - \\
Morningness-eveningness & - & - & - & - & - & - & - & - \\
\hline
\end{tabular}

$R^{2}$ the entire sample: 0.3 (explains $3 \%$ of the variance). $R^{2}$ males: 0.07 (explains $7 \%$ of the variance). $R^{2}$ females: 0.06 (explains $6 \%$ of the variance).

Table 6. Factors related to the level of cortisol $20 \mathrm{~h}$. Final stepwise, linear regression equation

\begin{tabular}{|c|c|c|c|c|c|c|c|c|c|}
\hline & \multicolumn{3}{|c|}{ Total sample } & \multicolumn{3}{|c|}{ Males } & \multicolumn{3}{|c|}{ Females } \\
\hline & $\beta$ & $t$ & $P$ & $\beta$ & $t$ & $p$ & $\beta$ & $t$ & $p$ \\
\hline Age & - & - & - & - & - & - & - & - & - \\
\hline Perceived health & - & - & - & - & - & - & - & - & - \\
\hline Mental health & - & - & - & - & - & - & - & - & - \\
\hline Job satisfaction & - & - & - & - & - & - & - & - & - \\
\hline Occupational stress & - & - & - & - & - & - & - & - & - \\
\hline Morningness-eveningness & -0.20 & 2.96 & 0.003 & -0.28 & -2.89 & 0.005 & - & - & - \\
\hline
\end{tabular}

$R^{2}$ the entire sample: 0.03 (explains 3\% of the variance). $R^{2}$ males: 0.07 (explains $7 \%$ of the variance). $R^{2}$ females: nonsignificant. 
stress. The psychological variables correlate more closely with occupational stress than with cortisol measured at $8 \mathrm{~h}$ and at $20 \mathrm{~h}$. In effect, as in the case of other variables such as overload or social support, the person's perception of the stressor (in this case, occupational stress) is more important than the "objective" measurement of stress through plasma cortisol. Previous studies [2] [9] have found the same tendency. In this work, when analyzing the regression equations for the entire sample, we found that psychological variables explained $16 \%$ of the variance of occupational stress versus 3\% of the variance explained by cortisol at $8 \mathrm{~h}$ and at $20 \mathrm{~h}$. When considering the three types of variables (occupational stress, cortisol at $8 \mathrm{~h}$ cortisol, and at $20 \mathrm{~h}$ ) separately, the psychological variables (perceived health, mental health, and job satisfaction) are more clearly associated with occupational stress (subjective measurement of stress).

Although linear differences in the target variables are minimal, the differential pattern of associations found among the stress variables is stronger when analyzing female and male nurses separately. In general, we found that slightly more variance of occupational stress (25\%) was explained by psychological variables than by cortisol at $8 \mathrm{~h}(7 \%)$ and cortisol at $20 \mathrm{~h}$ (7\%) in male nurses. In female nurses, 20\% of the variance of occupational stress was explained by psychological variables, $6 \%$ of the variance was explained by cortisol at 8 h, and no variance was explained by cortisol at $20 \mathrm{~h}$. This indicates that male nurses' stress is closely related to the psychological variables studied. To better clarify the differential pattern, we shall present the psychological variables associated with each type of stress measurement for the entire sample, and for males and females separately.

Perceived health and mental health are associated with occupational stress for the entire sample. Considering that, in both health measures, higher scores indicate poorer health, this means that greater occupational stress is related to poorer health. Age is the only variable associated with cortisol at $8 \mathrm{~h}$ in the entire sample; being younger is related to higher scores in cortisol at $8 \mathrm{~h}$. Cortisol at $20 \mathrm{~h}$ is associated with morningness-eveningness: higher morningness is related to higher scores in cortisol at $20 \mathrm{~h}$. This corresponds with the fact that the participants of the sample tended more towards eveningness than morningness, and were therefore more active in the afternoon (increased levels of cortisol in the afternoon [21].

In the group of male nurses, we also found perceived health and mental health associated with occupational stress and cortisol at $8 \mathrm{~h}$. As in the entire sample, higher occupational stress is related to worse self-perceived health and mental health. It is noteworthy that higher levels of occupational stress were related to lower cortisol at 8h; these results had been reported in other studies with professional nursing staff [5] [8]. Cortisol measured at $8 \mathrm{~h}$ in male nurses is also associated with poorer perceived health. As described for the entire sample, the association between cortisol at $20 \mathrm{~h}$ and morningness-eveningness was also observed in the males.

In the group of female nurses, we found an association between the variables age, perceived health and job satisfaction. In this case, being younger was related to worse self-perceived health, less job satisfaction, and more occupational stress. In cortisol at $8 \mathrm{~h}$, the only related variable was age; being younger was associated with higher cortisol scores. Perhaps these younger women are less experienced, feel more job insecurity, have more precarious contracts, and are more stressed because, due to their age, they may have small children to care for, etc. In this group, no associations between the target variables and cortisol at $20 \mathrm{~h}$ were found.

The only similarities found in the target variables and the measures of stress studied in the groups of males and females are worse self-perceived health and worse mental health associated with more occupational stress. The compositions of the remaining equations are different for male and female nurses, reinforcing the need to analyze data separately and not only to control for the variable age in the equations Sánchez-López, Cuellar-Flores, and Dresch [26].

Professional nursing staff, both male and female, are subject to high levels of occupational stress, partially explained by the very nature of the profession, that is, attending to and being in close contact with disease, pain, and death. Many studies have shown how occupational stress in male and female nurses is determined by work conditions (salary, work shifts, relations with coworkers, autonomy, and social acknowledgement) and personal situations (difficulty to conciliate family life and job, role overload, etc.), and, as shown in this study, occupational stress has a direct impact on health, both physical and mental. These results corroborate the need for the institutions to implement programs and action protocols to improve work conditions, and bring to the attention of the public these professionals' difficulties in their work and family settings and to stimulate their social and professional acknowledgement.

We would like to emphasize that this study of the topic is not exhaustive, and it presents some limitations. The first limitation was the nature of the sample studied. Because only nurses were analyzed, the results may not be representative of the general population. Second, this study was cross-sectional, and, therefore, we could not 
analyze changes produced in participants' over time or assess the temporal sequence of variables related to occupational stress and cortisol. Another limitation was the kind of measurement employed to evaluate physical health (Likert scale for self-perceived health without validation of medical records. Future studies could include objective measures and subjective of physical health to verify whether they coincide (e.g., laboratory tests that confirm diagnoses and diseases, immunological indicators, blood pressure, etc.).

\section{Acknowledgements}

Research funded by the Women’s Institute (Ministerio de Salud, Politica Social e Igualdad), reference number $18 / 09$.

We thank the Hospital Universitario de Getafe (Spain) for its involvement in the project.

\section{References}

[1] De la Banda, G.G., Martínez-Abascal, M.A., Riesco, M. and Pérez, G. (2004) La respuesta del cortisol ante un examen y su relación con otros acontecimientos estresantes y con algunas características de personalidad. Psicothema, 16, 294298.

[2] Almeida, D.M., Piazza, J.R. and Stawski, R.S. (2009) Interindividual Differences and Intraindividual Variability in the Cortisol Awakening Response: An Examination of Age and Gender. Psychology and Aging, 24, 918-827. http://dx.doi.org/10.1037/a0017910

[3] Kajantie, E. and Phillips, D.I.W. (2006) The Effects of Sex and Hormonal Status on the Physiological Response to Acute Psychosocial Stress. Psychoneuroendocrinology, 31, 151-178. http://dx.doi.org/10.1016/j.psyneuen.2005.07.002

[4] Deane, R., Chummun, H. and Prashad, D. (2002) Differences in Urinary Stress Hormones in Male and Female Nurses at Different Ages. Journal of Advanced Nursing, 37, 304-310. http://dx.doi.org/10.1046/j.1365-2648.2002.02082.x

[5] Harris, A., Ursin, H., Murison, R. and Eriksen, H.R. (2007) Coffee, Stress and Cortisol in Nursing Staff. Psychoneuroendocrinoly, 32, 322-330. http://dx.doi.org/10.1016/j.psyneuen.2007.01.003

[6] Kumari, M., Badrick, E., Chandola, T., Adam, E.K., Stafford, M., Marmot, M.G., Kirschbaum, C. and Kivimaki, M. (2009) Cortisol Secretion and Fatigue: Associations in a Community Based Cohort. Psychoneuroendocrinology, 34, 1476-1485. http://dx.doi.org/10.1016/j.psyneuen.2009.05.001

[7] Lindeberg, S.I., Eek, F., Lindbladh, E., Ostergren, P.-O., Hansen, A.M. and Karlson, B. (2008) Exhaustion Measured by the SF-36 Vitality Scale Is Associated with a Flattened Diurnal Cortisol Profile. Psychoneuroendocrinology, 33, 471-477. http://dx.doi.org/10.1016/j.psyneuen.2008.01.005

[8] Yang, Y., Koh, D., Ng, V., Lee, F.C.Y., Chan, G., Dong, F. and Chia, S.E. (2001) Salivary Cortisol Levels and WorkRelated Stress among Emergency Department Nurses. Journal of Occupational and Environmental Medicine, 43, 1011-1018. http://dx.doi.org/10.1097/00043764-200112000-00003

[9] Pruessner, J.C., Hellhammer, D.H. and Kirschbaum, C. (1999) Burnout, Perceived Stress, and Cortisol Responses to awAkening. Psychosomatic Medicine, 61, 197-204. http://dx.doi.org/10.3389/fpsyg.2015.00019

[10] Wang, J., Korczykowski, M., Rao, H., Fan, J., Pluta, J., Gur, R.C., McEwen, B.S. and Detre, J.A. (2007) Gender Difference in Neural Response to Psychological Stress. Social Cognitive \& Affective Neurosci, 2, 227-239. http://dx.doi.org/10.1093/scan/nsm018

[11] Kudielka, B.M. and Kirschbaum, C. (2005) Sex Differences in HPA Axis Responses to Stress: A Review. Biological Psychology, 69, 113-132. http://dx.doi.org/10.1016/j.biopsycho.2004.11.009

[12] Bergman, B., Ahmad, F. and Stewart, D.E. (2008) Work Family Balance, Stress, and Salivary Cortisol in Men and Women Academic Physicians. International Journal of Behavioral Medicine, 15, 54-61. http://dx.doi.org/10.1007/BF03003074

[13] Eriksen, W., Tambs, K. and Knardahl, S. (2006) Work Factors and Psychological Distress in Nurses’ Aides: A Prospective Cohort Study. BMC Public Health, 6, 290. http://dx.doi.org/10.1186/1471-2458-6-290

[14] Hsu, H., Chen, S., Yu, H. and Lou, J. (2010) Job Stress, Achievement Motivation and Occupational Burnout among Male Nurses. Journal of Advanced Nursing, 66, 1592-1601. http://dx.doi.org/10.1111/j.1365-2648.2010.05323.x

[15] Rochlen, A.B., Good, G.E. and Carver, T.A. (2009) Predictors of Gender-Related Barriers, Work, and Life Satisfaction among Men in Nursing. Psychology of Men \& Masculinity, 10, 44-56. http://dx.doi.org/10.1037/a0013291

[16] Polit, D.F. and Beck, C.T. (2009) International Gender Bias in Nursing Research, 2005-2006. International Journal of Nursing Studies, 46, 1102-1110. http://dx.doi.org/10.1016/j.ijnurstu.2009.02.002

[17] Ministerio de Sanidad y Consumo (2006) Encuesta Nacional de Salud. Ministerio de Sanidad y Consumo, Madrid. 
[18] Randler, C. and Schaal, S. (2010) Morningness-Eveningness, Habitual Sleep-Wake Variables and Cortisol Level. Biological Psychology, 85, 14-18. http://dx.doi.org/10.1016/j.biopsycho.2010.04.006

[19] Sánchez-López, M.P. and Dresch, V. (2008) The 12-Item General Health Questionnaire (GHQ-12): Reliability, External Validity and Factor Structure in the Spanish Population. Psicothema, 20, 839-843.

[20] Goldberg, D. and Williams, P. (1988) A User's Guide to the General Health Questionnaire. NFER-Nelson, Windsor, UK.

[21] Díaz-Morales, J.F. and Sánchez-López, M.P. (2005) Propiedades psicométricas de la Escala de Preferencias y la Escala Compuesta de Matutinidad [Psychometric Properties of Morningness Composite and Preferences Scales]. Psicothema, 17, 471-477.

[22] Smith, C., Reilly, C. and Midkiff, K. (1989) Evaluation of Three Circadian Rhythm Questionnaires with Suggestions for an Improved Measure of Morningness. Journal of Applied Psychology, 74, 728-738. http://dx.doi.org/10.1037/0021-9010.74.5.728

[23] Bartley, M. (2003) Health Inequality. An Introduction to Theories, Concepts and Methods. Polity Press, Cambridge, UK.

[24] Sánchez-López, M.P., Aparicio, M.E. and Dresch, V. (2006) Ansiedad, autoestima y satisfacción autopercibida como predictores la salud: Diferencias entre hombres y mujeres [Anxiety, Self-Esteem, and Self-Perceived Satisfaction as Predictors of Health: Differences between Men and Women]. Psicothema, 18, 584-590.

[25] Sánchez-López, M.P., López-García, J.J., Dresch, V. and Corbalán, J. (2008) Sociodemographic, Psychological and Health-Related Factors Associated with Poor Mental Health in Spanish Women and Men in Midlife. Women \& Health, 48, 445-465. http://dx.doi.org/10.1080/03630240802575096

[26] Sánchez-López, M.P., Cuellar-Flores, I. and Dresch, V. (2012) The Impact of Gender Roles on Health. Women \& Health, 52, 182-196. http://dx.doi.org/10.1080/03630242.2011.652352 